\title{
Research on the Propagation Mode of Network Self- Produced Variety Show Let's Talk Based on 5W Mode*
}

\author{
Lu Wang \\ Faculty of Arts and Sciences \\ Shanghai Polytechnic University \\ Shanghai, China 201209
}

\begin{abstract}
Let's Talk, a network self-produced debate program launched by $\mathrm{iQIYI}$, is a typical representative of network self-produced variety show in China. With accurate positioning and novel form, this program has broken the records of network self-produced variety show in financing, audience rating, advertising and other aspects, and its spreading influence cannot be underestimated. Based on the $5 \mathrm{~W}$ propagation mode, this paper makes an in-depth analysis of Let's Talk, and tries to show the prospect of this network self-produced variety show.
\end{abstract}

Keywords-network self-produced variety show; Let's Talk; $5 W$ propagation mode

\section{INTRODUCTION}

Let's Talk is a network self-produced debate program debuted in iQIYI, and a typical representative among China's network self-produced variety shows. The program has profound and extensive influence given the fact that it is accurate in positioning, novel in form, and has broken the records of network variety shows in financing, audience rating, advertising and other aspects. With the help of Lasswell's famous "5W" communication model: Who, Says What, In Which Channel, To Whom, and With What Effect, the propagation prospect of this network self-produced variety show can be depicted in a three-dimensional way.

\section{COMMUNICATOR}

As the leading role of the program, the communicator is an important factor in the program communication. There are four main types of communicators in Let's Talk: host, mentor, guest and contestant. Ma Dong serves as the host of the program, and the tutors will change slightly every season, but the main tutors are Gao Xiaosong and Cai Kangyong. In the fifth season, Li Dan, an Internet celebrity with high

*Fund: This paper is the research result of Shanghai Industrial University's Quality Curriculum Construction Project (code:

A01GY19G012-02); research results of the Public Relations Discipline Construction Project "New Media Public Relations Communication" of Shanghai Polytechnic University (discipline number: XXKPY 1610); and the achievement of the Special Plan for the Construction of the Teaching System of Curriculum Ideological and Political Education among Universities in Shanghai (code: A30DB191412-0214). traffic, and Xue Shaofeng, an economics teacher, joined the tutor group. The guests are selected based on popularity, and the contestants are selected through the auditions. The program production team firstly carries on the preliminary publicity for the program with the help of the celebrity effect brought by the host and the tutor. Second, the team also made use of the effect of popular guests participating in the recording to promote. These publicity means and celebrity effect further deepened the program publicity and influence.

A. Host

The host of Let's Talk is Ma Dong. As a host, Ma Dong plays the role of leading the program process and maximizing the program effect. At the beginning of the program, Ma Dong will introduce all the sponsored brands in due course. Before the debate, he will ask some small questions to interact with the tutor, and in the process, he will introduce the sponsored brands again in due course, without any feeling of awkward.

\section{B. Tutor}

The main regular tutors of Let's Talk are Gao Xiaoxong and Cai Kangyong. The tutor that joins newly are economist Xue Shaofeng, intellectual male Li Dan. The program selects people with rich life experience, humor, high EQ and high IQ to serve as mentors, and strives to bring new perspectives to the audience from different perspectives. The mentors in the program are not only the debaters who sometimes participate in the debate, but also the host and guests of the program. In each program, the mentor can choose a side that conforms to his or her own opinion and express his or her own opinion for this purpose.

\section{Guest}

Every two episodes of Let's Talk will invite a female guest or male guest to participate in the debate. For example, Yang Lan, Li Xiang, Xu Xiyuan (Hsu), Chen Handian, Xu Jinglei, Wu Jinyan and other popular stars. With the help of the topic and popularity of the guests, the audience will be attracted by the star effect, so as to improve the popularity of the program. It is not easy to choose the guests. The guests are the people with high traffic and popularity at present, 
which ensures the audience rating of the program and also increases the exposure of the guests. It is a mutually beneficial choice for both parties.

\section{Contestant}

The contestants in Let's Talk come from all walks of life and cover a wide range of identities. The contestants who can participate in the program through layers of selection are the most important soul part of the program. The debaters in the show are not as serious as those in traditional debate programs, and most of them need to conform to the characteristics of "weirdo", which means that the debater can express himself without restraint and hide himself. "Talk" means that the program seeks people who can speak. Debaters can show their true opinions and say what they want to say.

Different from traditional variety shows, Let's Talk is a free debate program, where debaters can show themselves and play freely. The show has no set script, and debaters can improvise and exude their enthusiasm.

\section{THE CONTENT OF COMMUNICATION}

\section{A. Debate Topics Based on Big Data}

As a variety debate program, the essential core of Let's Talk is debate, so the choice of debate topic has a crucial impact on the program. The topic of the debate should be close to the life of the public and be of interest to the public, so as to attract the attention of the audience and make the content of the program more influential. The topic of debate is not entirely selected by the program group. On Weibo, Zhihu (the Chinese Quora), Baidu Tieba and other platforms, the program selected some topics worthy discussion with big data, and then selected debate topic based on the results of netizens' votes. The weird and bizarre debate topics of Let's Talk completely breaks the old and uncreative topics of traditional programs, subverts the audience's outlook of the world, the life and the values, and enables the audience to view issue from multiple angles. It is quite different all other variety shows.

\section{B. The Language Symbol of the Program}

Program language, as a direct symbol, transmits program content and value, and influences the audiences. In addition to some weird and bizarre debate topics, the program also covers a lot of positive debate topics to provoke the audience to ponder. For example, one of the debates in the fifth season was titled do you support the idea that a dad who spends less than 12 hours a week with his children will be disqualified as a dad, which focuses on the widowed family education and arouses the audience's discussion and thinking. In addition, the language content of the subculture is also a major feature of the program.

As a variety show for young people, Let's Talk is full of subcultural language, with debates on "dirty" culture, homosexuality and other non-mainstream subcultures. Although the program claims to be "a serious debate program", it is serious about telling open "dirty" jokes, whose scale is slightly larger, but still within the acceptable range. It was evaluated by netizens as "agreeably greasy and dirty", which satisfied the audiences' repressed passion and the pleasure of fast free speech, and won the audience's love.

\section{The Non-verbal Symbols of the Program}

In order to meet its positioning for the generation after $90 \mathrm{~s}$, the program team has also made a lot of efforts in the visual and auditory nonverbal signs, and formed an exclusive style. The layout of the scene is bold in color, the stage background color is beautiful, and the modelling of the host and contestant is also very bold. Bright colors are used to show the vitality of young people and enhance the program's appeal. In terms of auditory sense, the post-production team used the exclusive special effects music as the opening sequence, and matched different sound effects in different contexts, which also added entertainment to the program. Bright colors, funny background sound effects, and lively atmosphere on the field, all make the program more interesting.

\section{CHANNELS OF COMMUNICATION}

As a new product of the Internet, the online variety show Let's Talk also combines the two-way interactive advantages of its own network transmission to expand the popularity of the program in addition to the traditional transmission mode.

\section{A. Online Promotion}

1) Exclusive play: Let's Talk is created by iQIYI and broadcast exclusively through iQIYI, the video website. IQIYI was founded by Baidu, one of the three Internet giants, with the company's philosophy of "Always Fun, Always Fine", and regard "User Experience" as highest goal. The audience can not only watch the program through the official website of the Internet, but also watch the program through mobile phones, tablet computers and other mobile terminals, ensuring that the online audience can easily watch the program anytime and anywhere.

2) Multi-media promotion: In the early stage of the program broadcast, the program team of Let's Talk not only uses traditional original media for advertising, but also complies with the trend of the times and made full use of big data to carry out targeted advertising campaigns on social softwares commonly used by the program audience. On the homepage screen of Weibo, WeChat public account, Douban, Zhihu and other social platforms as well as the floating window of the web page, the corresponding program publicity advertisements are carried out, so that the audience can immediately and directly notice the program publicity.

3) Interactive communication on social platforms: Weibo is the social platform that young people gather most and use most often. The program team of Let's Talk attaches importance to Weibo promotion for young audiences. The production team of Let's Talk sets up an official account on weibo, which is convenient for the promotion and 
online audiences and absorb their feedback. Online audiences can also initiate discussions about content. On social platforms, group communication is maximally applied to arouse the audience's group consciousness and resonance, and influence other individuals through the participation of the central audience in topic discussion, so as to promote the spread of the program and increase the popularity of the program.

\section{THE EFFECT OF COMMUNICATION}

\section{B. Offline Promotion}

In addition to online promotion, the program also carries out offline promotion. The program production team organizes auditions offline in the early stage of the program, excavates potential debaters and preheats up the program broadcast to some extent. In addition, the popular debaters' offline meeting held by the program group allows fans to ask questions on the spot and carry out some small games, so as to realize the zero-distance interaction between the communication subject and the audience and strengthen their internal connection.

\section{THE AUDIENCE}

\section{A. Analysis of Audience}

According to the data analysis of the second season of Let's Talk by Big Data Research Center, most Internet viewers of Let's Talk are female, accounting for 69\%, and most of them are young viewers aged 18-24. The production team of Let's Talk conducts audience analysis, audience positioning, audience contact and audience output through big data. Its precise audience positioning not only attracts the central audience to watch, but also influences the marginal audience, which further improves the program's ratings and attention.

In addition to the off-site audience, the live audience, as the most direct receiver of the program content, is selected by the production team according to the age level to experience the recording process. As the group that can feel the atmosphere of the on-site debate directly and have a deeper impression on the program, the audience can carry out the second transmission, so that the peers around them will be interested in the program.

\section{B. Analysis of Audience Behavior}

IQIYI has set up an official bullet screen for Let's Talk. The tutor and the contestant can tease it through bullet screen, which will increase the program feedback and help the program team to accept the feedback from the online audience. Online audiences can express their own opinions through the official bullet screen, or discuss program content with other audiences through bullet screen, which strengthens the participation of users and further deepens audiences' cohesion to the program, so as to fully meet the expression wishes of online audiences.

In addition, the production team of Let's Talk also uses the official Weibo account, WeChat official account and other social platforms to promote the program, interact with

\section{A. Attention Level: Young Audience Attention}

Attention level, as the first level of communication effect, is driven by initial shallow cognition. The production team of Let's Talk accurately targets the audience through big data, and makes targeted publicity on social platforms commonly used by the audience. The subculture in the bold, innovative and interesting program is what young audiences are interested in. The production team decides the debate topics and even the setting from the perspective of the young audience. The hot topics related to the content of the program have caused hot discussions on social platforms for many times, which are all due to the attention of young audiences and help to enhance the communication influence of the program.

\section{B. Emotional Level: Evoking Resonance and Concern}

As the second level of communication effect, the emotional level uses the same ideas and values to cause the emotional changes of the audience. Although an entertaining debate program cannot teach its audience debating skills, it can provide young audiences with life knowledge applicable to the current social life. In the process of program debate, debaters often use their own or other people's cases as proof to express their arguments, and use stories to arouse the resonance of the audience, change the audience's superficial cognition, deepen their thinking, so that audience can view problem from multiple perspectives, achieve deep cognition in the process of watching the debate, find the emotional resonance point through the expression of the debater, and relieve their inner emotions.

\section{Action Level: Mobilizing Audience Interaction}

As the third level of communication effect, the action level acts on the changes produced by the above two levels. Audience interaction of the program is embodied in three aspects: social platform interaction, real-time bullet screen feedback and participation in program recording. Online audiences forward and comment on the content of the program on social platforms, and post on the topic \#Let's Talk\# on Weibo to help the program group get relevant feedback. The program group also interacts with other audiences to expand the dissemination effect. When watching the program, the online audience can give feedback through real-time bullet screen. They can not only see the official bullet screen, but also see the information feedback from other audiences, which can more directly promote the program group to absorb opinions and improve and adjust the program production. In addition, audiences can 
participate in the live recording of the program, and audiences interested in the debate after watching the program can also participate in the selection through official registration channels. This is actually a two-way communication process. The audience promotes the spread of Let's Talk, and the program also attracts the audience to join the program recording.

\section{CONCLUSION}

As the benchmark of China's network self-produced variety shows, the successful program communication mode of Let's Talk is worthy of the industry practitioners' absorption and reference. Although the show's format is new and widely praised, there is still a lot of room for improvement, and the production team is also constantly improving and adjusting the content in the new season. In the future, practitioners should keep thinking about developing new ways to develop network self-produced variety shows. While making use of their own advantages to target the audience, they should focus on content, attach importance to originality, and create more excellent works in a standardized network environment.

\section{REFERENCES}

[1] CNNIC released the 42nd Statistical Report on the Development of China's Internet [R]. China Internet Network Information Center. 2018 (06). (in Chinese)

[2] Cai Xiaohua. Communication Interpretation of the Network Selfproduced Drama [J]. Culture \& Communication. 2013(06). (in Chinese)

[3] Liu Yang. Network Film and Television Transmission and Ontological Aesthetics in the Context of New Media [J]. Studies In National Art. 2010(05). (in Chinese)

[4] Big Data

Center. http://www.bigdata.ren/portal.php?mod=view\&aid=582

[5] Zhao Xiaolan. Ma Dong: Let "Qipa" Grow Wildly [J]. Global People. 2015(03) (in Chinese)

[6] Wang Tianyi. The Study on the Communication of Network Selfproduced Variety Show [D]. Guangxi University, 2017. (in Chinese) 